\title{
A Note on the Incompatibility of Strategy-Proofness and Pareto-Optimality in Quasi-Linear Settings with Public Budgets Working Paper
}

\author{
Ron Lavi` and Marina May ${ }^{\star \star}$ \\ Faculty of Industrial Engineering and Management \\ Technion, Israel Institute of Technology \\ ronlavi@ie.technion.ac.il, didra@techunix.technion.ac.il
}

\begin{abstract}
We study the problem of allocating multiple identical items that may be complements to budget-constrained bidders with private values. We show that there does not exist a deterministic mechanism that is individually rational, strategy-proof, Pareto-efficient, and that does not make positive transfers. This is true even if there are only two players, two items, and the budgets are common knowledge. The same impossibility naturally extends to more abstract social choice settings with an arbitrary outcome set, assuming players with quasi-linear utilities and public budget limits. Thus, the case of infinite budgets (in which the VCG mechanism satisfies all these properties) is really the exception.
\end{abstract}

* Supported in part by grants from the Israeli Science Foundation, the Bi-national Science Foundation, the Israeli ministry of science, and by the Google Inter-university center for Electronic Markets and Auctions.

** Supported in part by grants from the Israeli Science Foundation, the Bi-national Science Foundation, the Israeli ministry of science, and by the Google Inter-university center for Electronic Markets and Auctions. 